\title{
DEFORMING TRANSVERSE RIEMANNIAN METRICS OF FOLIATIONS*
}

\author{
MIROSLAV LOVRIĆ ${ }^{\dagger}$, MAUNG MIN-OO $\ddagger$, AND ERNST A. RUH ${ }^{\S}$
}

\begin{abstract}
The Ricci flow of Riemannian metrics on a compact manifold can be interpreted as a deformation of Cartan connections of hyperbolic type. The purpose of this paper is to show that there is analogous interpretation for the holonomy invariant transversal Riemannian metric defined for foliations over compact manifolds. Once the short-time existence of the transversal flow of Cartan connections is established, the theorems proved using deformation of the metric through the Ricci flow are generalized to the case of a Riemannian foliation on a compact Riemannian manifold.
\end{abstract}

1. Introduction and Results. In [9] the Ricci flow of Riemannian metrics on a compact manifold was interpreted as a deformation of Cartan connections of hyperbolic type. The purpose of this paper is to show that there is analogous interpretation for the holonomy invariant transversal Riemannian metric defined for foliations over compact manifolds.

As the first consequence, using the theorem of Hamilton [3, section 5] we obtain the short-time existence of the transversal flow of Cartan connections on $M$. Following the idea of Bemelmans, Min-Oo and Ruh introduced in [2] and using the maximum principle as in [1] we obtain the following smoothing property:

THEOREM 1.1. Let $\mathcal{F}$ be a foliation of codimension $q$ defined on a compact $n$ dimensional Riemannian manifold $M$ with a holonomy invariant transversal Riemannian metric $g_{Q}$ on the normal bundle $Q$. There exists a smooth curve of transversal Riemannian metrics $g(t)$ with $g(0)=g_{Q}$ and universal constants $C(q)$ and $\epsilon(q)$ depending only on the codimension $q$, such that

$$
\sum_{k=0}^{\infty} \frac{t^{k}}{((k+1) !)^{2}}\left|D^{k} F^{D}(g(t))\right|^{2} \leq C(q)\left|F^{D}(g(0))\right|^{2}
$$

uniformly for $t \in[0, \epsilon(q)]$, where $\left|D^{k} F^{D}(g(t))\right|$ is the supremum of the $k$-th covariant derivative of the transversal curvature tensor $F^{D}(g(t))$ of the metric $g(t)$.

Theorems proved using deformation of the metric through the Ricci flow (see $[7,8,12])$ can now be generalized to the case of a Riemannian foliation on a compact Riemannian manifold. In particular, we obtain the following theorems (see $[3,4]$ ):

THEOREM 1.2. Let $\mathcal{F}$ be a foliation of codimension three on a compact manifold $M$ with a holonomy invariant transversal Riemannian metric $g_{Q}$ for the normal bundle $Q$. Suppose that the Ricci curvature of $g_{Q}$ is positive definite. The metric $g_{Q}$ can be deformed to a holonomy invariant transversal Riemannian metric with constant positive sectional curvature.

*Received January 27, 1999; accepted for publication May 7, 1999.

†Department of Mathematics and Statistics, McMaster University, Hamilton, Ontario, Canada L8S 4K1 (lovric@icarus.math.mcmaster.ca). The research of this author was partially supported by Canadian NSERC Grant OGP-0194144.

$\ddagger$ Department of Mathematics and Statistics, McMaster University, Hamilton, Ontario, Canada L8S 4K1. The research of this author was partially supported by Canadian NSERC Grant OPG-7873.

$\S$ Department of Mathematics, University of Fribourg, Pérolles, CH-1700 Fribourg, Switzerland. The research of this author was partially supported by Swiss NSF grant \# 2000-50579.97. 
THEOREM 1.3. Let $\mathcal{F}$ be a foliation of codimension four on a compact manifold $M$ with a holonomy invariant transversal Riemannian metric $g_{Q}$ for the normal bundle $Q$. Suppose that the curvature operator of $g_{Q}$ is positive definite. The metric $g_{Q}$ can be deformed to a holonomy invariant transversal Riemannian metric with constant positive sectional curvature.

Similar result can be obtained in higher codimensions for a transversal curvature that is pinched enough (see $[7,8,12]$ ).

In order to prove theorems 1.2 and 1.3 we have to observe that once the existence of the flow (3.4) has been established, Hamilton's proof in [4] carries over to the more general setting of a Riemannian foliation since the Weitzenböck formulas are true locally on the local Riemannian quotient and because we can use the maximum principle for basic functions on the compact manifold exactly as it was done in [10]. The technical details will be dealt with in a forthcoming paper.

It is worth noting that the theorem of Hamilton concerning the Ricci flow on surfaces [5] does not have a generalization to Riemannian foliations. Technical reasons for this are the lack of a Gauss-Bonnet theorem and the non-existence of a suitable volume. A more basic reason is the counter-example of Hebda [6].

In Section 2 we present the background material and introduce Cartan connections. Section 3 is devoted to a study of the transversal flow of Cartan connections, which turns out to be the right tool - it enables us to extend the known results (see [4]) to foliations over Riemannian manifolds. In section 4 integrability and smoothness of the flow are established.

2. Main Concepts. Let $\left(M, g_{M}\right)$ be a compact Riemannian manifold and $\nabla^{M}$ its Levi-Civita connection. By $\mathcal{F}$ we denote a Riemannian foliation on $M$ defined by an integrable distribution $L^{p} \subseteq T M$ with normal bundle $Q^{q} \cong T M / L$.

The tangent bundle $T M$ splits orthogonally (with respect to $g_{M}$ ) as $T M=L \oplus L^{\perp}$, and $g_{M}=g_{L} \oplus g_{L^{\perp}}$. The metric $g_{Q}$ on $Q \cong L^{\perp}$ is defined as a pullback by local Riemannian submersions $f_{\alpha}$ (local transition functions are isometries) which describe the foliation $\mathcal{F}$. This metric is actually the pullback $\sigma^{*} g_{L^{\perp}}$, where $\sigma: Q \rightarrow L^{\perp} \subseteq T M$ splits the sequence

$$
0 \rightarrow L \rightarrow T M \stackrel{\mathrm{pr}_{Q}}{\longrightarrow} Q \rightarrow 0 .
$$

Such a metric satisfies [11, page 78] $L_{X} g_{Q}=0$ for $X \in \Gamma L$ and we say that the metric $g_{Q}$ is holonomy invariant (or that $g_{M}$ is bundle-like).

Basic Vector Fields and Basic Forms. A vector field $X$ defined on a domain $U \subseteq M$ is called basic if $X \in \Gamma Q$ (i.e. if $X$ is horizontal) and if $X$ is locally $f$-related to a vector field $\bar{X}$ defined on a local Riemannian quotient $f(U)$.

A vector field $X$ in $T M$ is called projectable if $[X, Y] \in \Gamma L$ whenever $Y \in \Gamma L$. (In local coordinates $X=\sum_{i=1}^{q} x_{i}\left(y_{1}, \ldots, y_{q}\right) \frac{\partial}{\partial y_{i}}$, where $\left\{y_{i}\right\}$ is the transversal coordinate frame). It is always possible to find a local orthonormal coordinate system $\left\{e_{1}, \ldots, e_{n}\right\}$ on $T M$ such that $e_{1}, \ldots, e_{p} \in \Gamma L$ and $e_{p+1}, \ldots, e_{n} \in \Gamma Q$ are projectable vector fields.

A differentiable form $\alpha$ on $M$ (with values in a vector bundle $E$ with connection $D$ ) is called basic iff $i_{X} \alpha=0$ and $L_{X} \alpha=0$ for $X \in \Gamma L$, or equivalently, iff both $\alpha$ and $d^{D} \alpha$ are horizontal. Such a form can be identified, on a distinguished chart $(U, f)$, with the pullback $\bar{\alpha}$ on the Riemannian quotient $f(U)$, i.e. $\alpha=f^{*} \bar{\alpha}$.

Basic Transversal Levi-Civita Connection in $Q$. The bundle $Q$ is canonically (independently of the metric) equipped with the partial connection (called the 
Bott connection)

$$
\nabla_{X}^{B} Y=\operatorname{pr}_{Q}\left[X, Y^{\prime}\right]
$$

where $X \in \Gamma L, Y \in \Gamma Q$ and $Y^{\prime} \in \Gamma T M$ is such that $\operatorname{pr}_{Q}\left(Y^{\prime}\right)=Y$. This connection is flat (i.e. $R^{\nabla^{B}}(X, Y)=0$ whenever $\left.X, Y \in \Gamma L\right)$ and $\nabla_{X}^{B} Y=0$ for a basic vector field $Y$. It is not the restriction of the Levi-Civita connection $\nabla^{M}$ of $T M$ to $L$.

To extend the Bott connection we define

$$
\nabla_{X} Y=\operatorname{pr}_{Q}\left(\nabla_{X}^{M} Y^{\prime}\right)
$$

for $X, Y \in \Gamma Q$, and $Y^{\prime} \in \Gamma T M$ is such that $\operatorname{pr}_{Q}\left(Y^{\prime}\right)=Y$.

The connection $\nabla_{X} Y$ for $X \in \Gamma T M$ and $Y \in \Gamma Q$ defined by (2.2) and (2.3) is called adapted to Bott connection $\nabla_{X}^{B} Y$. It is metric (with respect to $g_{Q}$ ) [13, page 53], and torsion free [13, page 49] and is called the transversal Levi-Civita connection of $\mathcal{F}$. It coincides (because of local uniqueness) with the pullback of the local LeviCivita connection on the Riemannian quotient. Such a connection is called basic. Equivalent statement, $i_{X} R^{\nabla}=0$ for $X \in \Gamma L$, is a consequence of the Jacobi identity.

Gauge Transformations. Any invertible endomorphism $\theta: Q \rightarrow Q$ can be extended to a homomorphism $\theta=\theta \circ \operatorname{pr}_{Q}: T M \rightarrow Q$, where $\operatorname{pr}_{Q}: T M \rightarrow Q$ is a projection onto $Q$ defined using the (fixed) metric $g_{M}$ on $M$. It can be easily verified that if $L_{X} \theta=0$ for $X \in \Gamma L$, then $\theta$ is a basic 1 -form on $M$ with values in the bundle $Q$.

The gauge transform of a connection $\nabla$ in $Q$ is $\left(\theta^{*} \nabla\right)_{X} Y=\theta^{-1}\left(\nabla_{X}(\theta Y)\right)$. The curvature is then $\theta^{*} R(X, Y)(Z)=\theta^{-1}(R(X, Y)(\theta Z))$.

The gauge transform of the metric $g_{Q}$ in $Q$ is $\theta^{*} g_{Q}(X, Y)=g_{Q}(\theta X, \theta Y)$, where $X, Y \in \Gamma Q$.

Cartan Connections. Consider a vector bundle $E=Q \oplus Q \wedge Q$ over a compact manifold $M$. The bundle $Q \wedge Q$ is identified (via $g_{Q}$ ) with the bundle of skewsymmetric endomorphisms of $Q$, i.e.

$$
(X \wedge Y) \leftrightarrow\left(Z \mapsto g_{Q}(Z, Y) X-g_{Q}(Z, X) Y\right) .
$$

The fibers of $E$ are isomorphic to $o(1, n)$, and the Lie algebra structure is given by

$$
[(X, A),(Y, B)]=\left(A(Y)-B(X),[A, B]_{Q \wedge Q}+X \wedge Y\right),
$$

where $X, Y \in \Gamma Q$ and $A, B \in \Gamma(Q \wedge Q)$.

The metric $g_{E}$ in fibers of $E$ is a direct sum metric induced by the base metric $g_{M} ;$ hence $g_{E}(A, B)=-\frac{1}{2} \operatorname{tr}(A B)$ and $g_{E}(A, X \wedge Y)=-g_{M}(A(X), Y)$ for $X, Y \in \Gamma Q$ and skew-symmetric maps $A, B: Q \rightarrow Q$.

Combining the connection on $E=Q \oplus Q \wedge Q$ induced by $\nabla$ (see definitions (2.2) and (2.3)) with the gauge transform $\theta$ we form a Cartan connection of the type $o(1, n)$

$$
D_{X} s=\nabla_{X} s+[\theta X, s]
$$

where $X \in \Gamma T M, s \in \Gamma E$ and the bracket being defined in (2.5). Since both $\nabla$ and $\theta$ are basic it follows that the Cartan connection $D$ is also basic, i.e. is a local lift of a Cartan connection on a local Riemannian quotient. 
Take any $\alpha \in \Omega^{p}(M ; E)$. The exterior covariant derivative with respect to the connection $D$ is defined by

$$
\begin{gathered}
d^{D} \alpha\left(X_{0}, \ldots, X_{p}\right)=\sum_{i=0}^{n}(-1)^{i} D_{X_{i}}\left(\alpha\left(X_{0}, \ldots, \widehat{X_{i}}, \ldots, X_{p}\right)\right) \\
+\sum_{1 \leq i<j}^{n}(-1)^{i+j} \alpha\left(\left[X_{i}, X_{j}\right], X_{0}, \ldots, \widehat{X}_{i}, \ldots, \widehat{X}_{j}, \ldots, X_{p}\right),
\end{gathered}
$$

where $X_{0}, \ldots, X_{p} \in \Gamma T M$. The exterior derivative $d^{\nabla}$ with respect to the connection $\nabla$ on $Q$ adapted to the Bott connection is defined analogously. Hence

$$
d^{D} \alpha=d^{\nabla} \alpha+d_{2} \alpha
$$

where $d_{2}$ is the algebraic operator

$$
d_{2} \alpha\left(X_{0}, \ldots, X_{p}\right)=\sum_{i=0}^{n}(-1)^{i}\left[\theta\left(X_{i}\right), \alpha\left(X_{0}, \ldots, \widehat{X}_{i}, \ldots, X_{p}\right)\right] .
$$

Let $F^{D}$ be the curvature of the connection $D, F^{D} \in \Omega^{2}\left(M ; E^{*} \otimes E\right)$. A simple computation shows that

$$
F^{D}(X, Y) s=R^{\nabla}(X, Y) s+[\theta X \wedge \theta Y, s]+\left[d^{\nabla} \theta(X, Y), s\right]
$$

for $X, Y \in \Gamma T M$ and $s \in \Gamma E$. Therefore the curvature $F^{D}$ is the direct sum $F^{D}=$ $F_{1}+F_{2}$, where $F_{1}$ has values in $Q \wedge Q$ and is given by

$$
F_{1}(X, Y)=R^{\nabla}(X, Y)+\theta X \wedge \theta Y
$$

and

$$
F_{2}(X, Y)=d^{\nabla} \theta(X, Y)
$$

has values in $Q$. The Cartan torsion is defined by the formula

$$
T^{\nabla, \theta}(X, Y)=F_{2}(X, Y)=\nabla_{X}(\theta Y)-\nabla_{Y}(\theta X)-\theta([X, Y]) .
$$

Lemma 2.1 (Bianchi identities). Assume that the Cartan torsion is zero. Then $d_{2} F=0$ and $d^{\nabla} F=0$.

Proof. A straightforward computation gives

$$
\begin{aligned}
d_{2} & F_{1}(X, Y, Z)=\left[\theta X, F_{1}(Y, Z)\right]-\left[\theta Y, F_{1}(X, Z)\right]+\left[\theta Z, F_{1}(X, Y)\right] \\
= & -F_{1}(Y, Z) \theta X+F_{1}(X, Z) \theta Y-F_{1}(X, Y) \theta Z \\
= & -R^{\nabla}(Y, Z) \theta X-g_{Q}(\theta X, \theta Z) \theta Y+g_{Q}(\theta X, \theta Y) \theta Z \\
& +R^{\nabla}(X, Z) \theta Y+g_{Q}(\theta Y, \theta Z) \theta X-g_{Q}(\theta Y, \theta X) \theta Z \\
& -R^{\nabla}(X, Y) \theta Z-g_{Q}(\theta Z, \theta Y) \theta X+g_{Q}(\theta Z, \theta X) \theta Y \\
= & -\theta\left(\theta^{-1} R^{\nabla}(Y, Z) \theta X+\theta^{-1} R^{\nabla}(Z, X) \theta Y+\theta^{-1} R^{\nabla}(X, Y) \theta Z\right) \\
= & -\theta\left(R^{\theta^{*} \nabla}(Y, Z)(X)+R^{\theta^{*} \nabla}(Z, X)(Y)+R^{\theta^{*} \nabla}(X, Y)(Z)\right)=0
\end{aligned}
$$

by the first Bianchi identity. Furthermore,

$$
d^{\nabla} F_{1}=d^{\nabla} R^{\nabla}+d^{\nabla}(\theta \wedge \theta)=0,
$$

since $d^{\nabla} R^{\nabla}=0$, and $d^{\nabla} \theta=0$ by assumption. 
3. Transversal Flow of Cartan Connections. In this section we consider Cartan connections $D=\nabla+\theta$ on a vector bundle $E=Q \oplus Q \wedge Q$ satisfying the following requirements:

(i) $\nabla$ restricts to the Bott connection $\nabla^{B}$ on $\Gamma L$

(ii) $\nabla g_{Q}=0$, where $g_{Q}$ is the (fixed) initial transversal metric, and

(iii) the Cartan torsion $T^{\nabla, \theta}=0$.

Lemma 3.1. The connection $\theta^{*} \nabla$ is the basic Levi-Civita connection of the transversal Riemannian metric $\theta^{*} g_{Q}$.

Proof. Since

$$
\begin{aligned}
& \left(\left(\theta^{*} \nabla\right)_{U}\left(\theta^{*} g_{Q}\right)\right)(X, Y) \\
& =U\left(\theta^{*} g_{Q}(X, Y)\right)-\theta^{*} g_{Q}\left(\left(\theta^{*} \nabla\right)_{U} X, Y\right)-\theta^{*} g_{Q}\left(X,\left(\theta^{*} \nabla\right)_{U} Y\right) \\
& =U\left(g_{Q}(\theta X, \theta Y)\right)-g_{Q}\left(\nabla_{U}(\theta X), \theta Y\right)-g_{Q}\left(X, \nabla_{U}(\theta Y)\right)=0
\end{aligned}
$$

and

$$
\begin{aligned}
& T^{\theta^{*} \nabla}(X, Y)=\left(\theta^{*} \nabla\right)_{X} \operatorname{pr}_{Q}(Y)-\left(\theta^{*} \nabla\right)_{Y} \operatorname{pr}_{Q}(X)-\operatorname{pr}_{Q}[X, Y] \\
& =\theta^{-1} \nabla_{X}(\theta Y)-\theta^{-1} \nabla_{Y}(\theta X)-\theta^{-1}(\theta[X, Y]) \\
& =\theta^{-1}\left(T^{\nabla, \theta}(X, Y)\right)=0
\end{aligned}
$$

it follows that $\theta^{*} \nabla$ is the Levi-Civita connection of $\theta^{*} g_{Q}$. The fact that it is basic follows from

$$
\left(i_{X} R^{\theta^{*} \nabla}\right)(Y)=R^{\theta^{*} \nabla}(X, Y)=\theta^{-1}\left(R^{\nabla}(X, Y) \theta\right)
$$

and the fact that $\nabla$ is basic.

Consider the flow of Cartan connections $D=\nabla+\theta$

$$
\frac{\partial}{\partial t} D=-\tilde{\delta}^{D} F^{D}
$$

on a vector bundle $E=Q \oplus Q \wedge Q$, with the initial condition $D(0)=\nabla+$ id. The operator $\tilde{\delta}^{D}$ is "almost" adjoint of the exterior covariant derivative $d^{D}$, and is given by the formula

$$
\begin{aligned}
& \tilde{\delta}^{D} \alpha\left(X_{2}, \ldots, X_{p}\right)=-\sum_{k=1}^{n} \nabla_{e_{k}}\left(\alpha\left(e_{k}, X_{2}, \ldots, X_{p}\right)\right) \\
& +\sum_{k=1}^{n} \alpha\left(\tilde{\nabla}_{e_{k}} e_{k}, X_{2}, \ldots, X_{p}\right)+\sum_{k=1}^{n} \sum_{j=2}^{n} \alpha\left(e_{k}, X_{2}, \ldots, \tilde{\nabla}_{e_{k}} X_{j}, \ldots, X_{p}\right) \\
& +\sum_{k=1}^{n}\left[\theta e_{k}, \alpha\left(e_{k}, X_{2}, \ldots, X_{p}\right)\right]
\end{aligned}
$$

where $X_{2}, \ldots, X_{p} \in \Gamma T M,\left\{e_{k}\right\}$ is an orthonormal basis of $T M$ for the metric $g_{L} \oplus \theta^{*} g_{Q}$ and $\tilde{\nabla}$ is the metric connection (which preserves the splitting $T M=L \oplus Q$ ) given by

$$
\tilde{\nabla}_{X} Y=\operatorname{pr}_{L} \nabla_{X}^{M}\left(\operatorname{pr}_{L} Y\right)+\operatorname{pr}_{Q} \nabla_{X}^{M}\left(\operatorname{pr}_{Q} Y\right)
$$


for $X, Y \in \Gamma T M$. The connection $\nabla^{M}$ in (3.6) is the Levi-Civita connection of the metric $g_{M}$ on $M$ and $\operatorname{pr}_{L}$ the projection $T M \rightarrow L$.

The operator $\tilde{\delta}^{D}$ leaves basic forms invariant and coincides, on a distinguished chart $U$, with the lift of the usual formal adjoint on the local Riemannian quotient $f(U)$ (see [10, Prop. 2.6]).

Define the operators $\delta_{2}$ and $\tilde{\delta}^{\nabla}$ by:

$$
\delta_{2} \alpha\left(X_{2}, \ldots, X_{p}\right)=\left[\theta e_{k}, \alpha\left(e_{k}, X_{2}, \ldots, X_{p}\right)\right]
$$

and

$$
\begin{aligned}
& \tilde{\delta}^{\nabla} \alpha\left(X_{2}, \ldots, X_{p}\right)=-(\tilde{\nabla} \otimes \nabla)_{e_{k}} \alpha\left(e_{k}, X_{2}, \ldots, X_{p}\right) \\
& =-\sum_{k=1}^{n} \nabla_{e_{k}}\left(\alpha\left(e_{k}, X_{2}, \ldots, X_{p}\right)\right)+\sum_{k=1}^{n} \alpha\left(\tilde{\nabla}_{e_{k}} e_{k}, X_{2}, \ldots, X_{p}\right) \\
& +\sum_{k=1}^{n} \sum_{j=2}^{n} \alpha\left(e_{k}, X_{2}, \ldots, \tilde{\nabla}_{e_{k}} X_{j}, \ldots, X_{p}\right)
\end{aligned}
$$

for a p-form $\alpha$ on $M$ with values in $E, X_{2}, \ldots, X_{p} \in \Gamma T M$ and a frame $\left\{e_{k}\right\}$ of $T M$ orthonormal with respect to the metric $g_{L} \oplus \theta^{*} g_{Q}$. The operator $\tilde{\delta}^{D}$ defined in (3.5) can now be written as

$$
\tilde{\delta}^{D} \alpha=\tilde{\delta}^{\nabla} \alpha+\delta_{2} \alpha
$$

The Ricci tensor, viewed as a 1 -form with values in $T M$ can be expressed as

$$
\operatorname{Ric}(X)=R\left(X, e_{k}\right) e_{k}=\left[e_{k}, R\left(e_{k}, X\right)\right]
$$

where the full curvature $R$ is interpreted as a 2 -form with values in $T M \wedge T M$.

The short-time existence of the flow (3.4) is proved in the next section.

Lemma 3.2. Let $F_{2}=0$. Then $\dot{\nabla}=-\tilde{\delta}^{\nabla} F$ and $\dot{\theta}=-\delta_{2} F$.

Proof. By definition of the Cartan connection it follows that

$$
\dot{D}_{X} s=\dot{\nabla}_{X} s+[\dot{\theta} X, s]
$$

since the Lie algebra structure in fibers is fixed. On the other hand, by the definition (3.5)

$$
\left(\tilde{\delta}^{D} F(X)\right) s=\left(\tilde{\delta}^{\nabla} F(X)\right) s+\left[\delta_{2} F(X), s\right] .
$$

Comparison of the components of $Q$ and $Q \wedge Q$ in (3.4) and the fact that $F_{2}=0$ imply the statement of the lemma.

LEMma 3.3. Let $F_{2}=0$. The flow (3.4) induces the following evolution equation for the transversal metric:

$$
\dot{g}_{Q}(X, Y)=-2 g_{Q}\left(\operatorname{Ric}\left(g_{Q}\right)(X), Y\right)-2(n-1) g_{Q}(X, Y) .
$$


Proof. By the previous lemma the time derivative of the (changing) metric $\theta^{*} g_{Q}$ is computed to be

$$
\begin{aligned}
& \frac{\partial}{\partial t}\left(\theta^{*} g_{Q}(X, Y)\right)=g_{Q}(\dot{\theta} X, \theta Y)+g_{Q}(\theta X, \dot{\theta} Y) \\
& =-g_{Q}\left(\left[\theta e_{k}, F\left(e_{k}, X\right)\right], \theta Y\right)-g_{Q}\left(\left[\theta e_{k}, F\left(e_{k}, Y\right)\right], \theta X\right)
\end{aligned}
$$

for a $\theta^{*} g_{Q}$-orthonormal basis $\left\{e_{k}\right\}$. Now since $F_{2}=0$,

$$
\begin{aligned}
g_{Q} & \left(\left[\theta e_{k}, F\left(e_{k}, X\right)\right], \theta Y\right)=g_{Q}\left(-F_{1}\left(e_{k}, X\right) \theta e_{k}, \theta Y\right) \\
= & -g_{Q}\left(R^{\nabla}\left(e_{k}, X\right) \theta e_{k}, \theta Y\right)-g_{Q}\left(\left(\theta e_{k} \wedge \theta X\right) \theta e_{k}, \theta Y\right) \\
= & -\theta^{*} g_{Q}\left(R^{\theta^{*} \nabla}\left(e_{k}, X\right) e_{k}, Y\right)-g_{Q}\left(\theta e_{k}, \theta X\right) g_{Q}\left(\theta e_{k}, \theta Y\right) \\
& +g_{Q}\left(\theta e_{k}, \theta e_{k}\right) g_{Q}(\theta X, \theta Y) \\
= & \theta^{*} g_{Q}\left(\operatorname{Ric}^{\theta^{*} \nabla}(X), Y\right)+(n-1) \theta^{*} g_{Q}(X, Y) .
\end{aligned}
$$

The result now follows from the fact that (3.14) is symmetric in $X$ and $Y$. $\square$

Equation (3.13) differs from Hamilton's [3, page 259] in the normalizing factor.

LemMa 3.4. Let $F_{2}=0$. Then $\dot{\nabla}_{V} X=0$ for $V \in \Gamma L$ and $X \in \Gamma Q$.

Proof. The statement follows from the equation $\dot{\nabla}_{V} X=-\left(\tilde{\delta}^{\nabla} F(V)\right)(X)$ (see lemma 3.2 ) and the fact that $F=F_{1}$ is basic. $\square$

LeMma 3.5. Let $F_{2}=0$. Then $\dot{F}_{2}=-d_{2} \tilde{\delta}^{\nabla} F-d^{\nabla} \delta_{2} F$.

Proof. A straightforward computation gives

$$
\begin{aligned}
& \dot{F}_{2}(X, Y)=\dot{\nabla}_{X}(\theta Y)-\dot{\nabla}_{Y}(\theta X)+\nabla_{X}(\dot{\theta} Y)-\nabla_{Y}(\dot{\theta} X)-\dot{\theta}[X, Y] \\
& =-\tilde{\delta}^{\nabla} F_{1}(X)(\theta Y)+\tilde{\delta}^{\nabla} F_{1}(Y)(\theta X)+d^{\nabla} \dot{\theta}(X, Y) \\
& =\left[\theta Y, \tilde{\delta}^{\nabla} F(X)\right]-\left[\theta X, \tilde{\delta}^{\nabla} F(Y)\right]+d^{\nabla} \dot{\theta}(X, Y) \\
& =-d_{2} \tilde{\delta}^{\nabla} F(X, Y)-d^{\nabla} \delta_{2} F(X, Y),
\end{aligned}
$$

by lemma 3.2 and definition (2.9).

Now we show that the flow (3.4) is tangent to the space of Cartan connections with vanishing Cartan torsion.

LEMMA 3.6. Let $F_{2}=0$. Then $\dot{F}_{2}=0$.

Proof. Let $\left\{e_{k}\right\}$ be an orthonormal basis of $T M$ with respect to the metric $g_{L} \oplus \theta^{*} g_{Q}$ chosen so that $e_{1}, \ldots, e_{p} \in \Gamma L$ and $e_{p+1}, \ldots, e_{n} \in \Gamma Q$ are projectable vector fields. Take $X=X_{L}+X_{Q}$ and $Y=Y_{L}+Y_{Q}$ in $T M$ with transversal components $X_{Q}$ and $Y_{Q}$ being projectable vector fields (such a choice suffices because (3.16) is linear with respect to smooth functions on $M$ ). Since $\nabla^{M}$ is the Levi-Civita connection, we can assume that at the point $x$ on $M$ the vector field bracket and the covariant derivatives of vectors $e_{k}, X_{L}, X_{Q}, Y_{L}$ and $Y_{Q}$ vanish.

If $e_{k} \in \Gamma L$ then $F\left(e_{k}, \tilde{\nabla}_{e_{k}} X\right)=0$ since $F=F_{1}$ is basic and $F\left(\tilde{\nabla}_{e_{k}} e_{k}, X\right)=0$ since $\tilde{\nabla}_{e_{k}} e_{k}=\operatorname{pr}_{L}\left(\nabla_{e_{k}}^{M} e_{k}\right)=0$. If $e_{k} \in \Gamma Q$ then $\tilde{\nabla}_{e_{k}} e_{k}=\operatorname{pr}_{Q}\left(\nabla_{e_{k}}^{M} e_{k}\right)=0$ and $\tilde{\nabla}_{e_{k}} X=0$ by the choice of $e_{k}$ and $X$ and by definition (3.6) of the connection $\tilde{\nabla}$ on $M$. In any case,

$$
\tilde{\delta}^{\nabla} F(X)=-\nabla_{e_{k}} F\left(e_{k}, X\right)=-\nabla_{e_{k}}\left(F\left(e_{k}, X\right)\right)
$$


Definitions (2.2) and (2.3) and the assumption $\nabla^{M} X_{Q}=0$ imply that $\nabla X_{Q}=0$. The fact that the gauge transform $\theta^{-1} \nabla \theta$ of $\nabla$ is basic implies that their projections on the local Riemannian quotient are also related by the gauge transform $\theta$ and therefore, since $\nabla X_{Q}=0$, it follows that $\theta^{-1} \nabla \theta X_{Q}=0$. Consequently, $\nabla \theta X=0$ $\left(\nabla \theta X_{L}=0\right.$ holds by the definition of $\left.\theta\right)$ and analogously $\nabla \theta Y=0$ and $\nabla \theta e_{k}=0$. This consideration simplifies the following computations (see Lemma 7 in [9]):

$$
\begin{aligned}
& d_{2} \tilde{\delta}^{\nabla} F(X, Y)=\left[\theta X, \tilde{\delta}^{\nabla} F(Y)\right]-\left[\theta Y, \tilde{\delta}^{\nabla} F(X)\right] \\
& =-\left[\theta X, \nabla_{e_{k}}\left(F\left(e_{k}, Y\right)\right)\right]+\left[\theta Y, \nabla_{e_{k}}\left(F\left(e_{k}, X\right)\right)\right] \\
& =\nabla_{e_{k}}\left(\left[\theta X, F\left(Y, e_{k}\right)\right]+\left[\theta Y, F\left(e_{k}, X\right)\right]\right) \\
& =-\nabla_{e_{k}}\left[\theta e_{k}, F(X, Y)\right],
\end{aligned}
$$

by the Bianchi identity $d_{2} F=0$. On the other hand

$$
\begin{aligned}
& d^{\nabla} \delta_{2} F(X, Y)=\nabla_{X}\left(\delta_{2} F(Y)\right)-\nabla_{Y}\left(\delta_{2} F(X)\right) \\
& =\nabla_{X}\left(\left[\theta e_{k}, F\left(e_{k}, Y\right)\right]\right)-\nabla_{Y}\left(\left[\theta e_{k}, F\left(e_{k}, X\right)\right]\right) \\
& =\left[\theta e_{k}, \nabla_{X}\left(F\left(e_{k}, Y\right)\right)-\nabla_{Y}\left(F\left(e_{k}, X\right)\right)\right] \\
& =\nabla_{e_{k}}\left[\theta e_{k}, F(X, Y)\right],
\end{aligned}
$$

by the Bianchi identity $d^{\nabla} F=0$.

4. Integrability and Smoothness of the Flow. Our next goal is to prove the short time integrability of the flow (3.4). To avoid introducing additional notation we will use the symbol $g_{Q}$ to denote the metric on $E=Q \oplus Q \wedge Q$ induced by the metric $g_{Q}$ on $Q$. Fix the connection $D(0)$ on $E$. Any other connection $D$ on $E$ can be expressed as $D=D(0)+A$ with $A \in \Omega^{1}\left(M ; E^{*} \otimes E\right)$, and therefore we can rewrite (3.4) as the flow

$$
\frac{\partial}{\partial t} A=-\tilde{\delta}_{\theta^{*} g_{Q}}^{D(0)+A} F^{D(0)+A}
$$

in $\Omega^{1}\left(M ; E^{*} \otimes E\right)$ with initial condition $A=0$. The symbol $\tilde{\delta}_{\theta^{*} g_{Q}}^{D(0)+A}$ denotes the adjoint (3.5) defined with respect to the connection $D(0)+A$ and the gauge-transformed metric $\theta^{*} g_{Q}$.

Proposition 4.1. The evolution equation (4.1) has a unique smooth solution for some (small) time interval $[0, T>0]$ for any initial condition $D(0)$.

Proof. The time derivative of $A$ is computed to be

$$
\begin{aligned}
& \frac{\partial}{\partial t} A=-\tilde{\delta}_{\theta^{*} g_{Q}}^{D(0)+A} F^{D(0)+A} \\
& =\left(-\tilde{\delta}_{g_{Q}}^{D(0)} \cdot+A \vee \cdot-G \cdot\right)\left(F^{D(0)}+d^{D(0)} A+[A, A]\right) \\
& =-\tilde{\delta}_{g_{Q}}^{D(0)} d^{D(0)} A-\tilde{\delta}_{g_{Q}}^{D(0)}[A, A]+A \vee d^{D(0)} A-G \cdot d^{D(0)} A \\
& \quad+A \vee F^{D(0)}+A \vee[A, A]-G \cdot[A, A]-\tilde{\delta}_{g_{Q}}^{D(0)} F^{D(0)}-G \cdot F^{D(0)}
\end{aligned}
$$

where

$$
\tilde{\delta}_{g_{Q}}^{D(0)} \alpha\left(X_{2}, \ldots, X_{p}\right)=-(\tilde{\nabla} \otimes D(0))_{e_{k}} \alpha\left(e_{k}, X_{2}, \ldots, X_{p}\right)
$$


and $\left\{e_{k}\right\}$ is a $g_{Q}$-orthonormal frame of $T M, d^{D(0)}$ is the exterior derivative operator defined in (2.7),

$$
A \vee \alpha\left(X_{2}, \ldots, X_{p}\right)=\sum_{k=1}^{n} A\left(e_{k}\right) \alpha\left(e_{k}, X_{2}, \ldots, X_{p}\right)
$$

and

$$
\begin{aligned}
& G . \alpha\left(X_{2}, \ldots, X_{p}\right)=\sum_{k=1}^{n} \alpha\left(G\left(e_{k}\right) e_{k}, X_{2}, \ldots, X_{p}\right) \\
& +\sum_{k=1}^{n} \sum_{j=2}^{p} \alpha\left(e_{k}, X_{1}, \ldots, G\left(e_{k}\right) X_{j}, \ldots, X_{p}\right) \\
& +\sum_{k=1}^{n} G_{k}(\theta, \partial \theta) \alpha\left(e_{k}, X_{2}, \ldots, X_{p}\right)
\end{aligned}
$$

where $G_{k}$ are smooth functions in $\theta$ and its partial derivatives and

$$
G(X) Y=\theta^{-1}\left(\tilde{\nabla}_{X}(\theta Y)-\theta \tilde{\nabla}_{X} Y\right)=\left(\theta^{-1} \circ\left(\tilde{\nabla}_{X} \theta\right)\right) Y
$$

for $X, Y \in \Gamma T M$. Hence

$$
\frac{\partial}{\partial t} A=-\tilde{\delta}_{g_{Q}}^{D(0)} d^{D(0)} A+\text { terms of lower order in A, }
$$

with the second order term given by

$$
-\tilde{\delta}_{g_{Q}}^{D(0)} d^{D(0)} A(X)=-\sum_{k=1}^{n} D(0)_{e_{k}} D(0)_{e_{k}} A(X)-\sum_{k=1}^{n} D(0)_{e_{k}} D(0)_{X} A\left(e_{k}\right)
$$

$$
\text { +lower order terms. }
$$

Define the operators

$$
L(A) H=\tilde{\delta}_{\theta^{*} g_{Q}}^{D(0)+A} H
$$

for $H \in \Omega^{1}\left(M ; E^{*} \otimes E\right)$, and

$$
E(A)=-\tilde{\delta}_{\theta^{*} g_{Q}}^{D(0)+A} F^{D(0)+A} .
$$

Now $L(A) E(A)=-\left(\tilde{\delta}_{\theta^{*} g_{Q}}^{D(0)+A}\right)^{2}$ is a first-order operator, since $\tilde{\delta}$ is adjoint to $d$ up to an algebraic term, see (3.5).

From (4.8) it follows that the symbol of the derivative $\mathrm{DE}(\mathrm{A})$ in the cotangent direction $\xi$ equals

$$
(\sigma D E(A)(\xi) H)_{j}=\left(\sum_{k=1}^{n} \xi_{k}^{2}\right) H_{j}-\sum_{k=1}^{n} \xi_{k} \xi_{j} H_{k}
$$

The symbol of $L(A)$ is given by

$$
\sigma L(A)(\xi) H=-\sum_{k=1}^{n} \xi_{k} H_{k}
$$


and therefore the nullspace $N$ of $\sigma L(A)(\xi)$ consists of all $H \in \Omega^{1}\left(M ; E^{*} \otimes E\right)$ satisfying $\sum_{k=1}^{n} \xi_{k} H_{k}=0$. The restriction of $\sigma D E(A)(\xi)$ to $N$ is then

$$
\left(\left.\sigma D E(A)(\xi)\right|_{N} H\right)_{j}=\left(\sum_{k=1}^{n} \xi_{k}^{2}\right) H_{j},
$$

i.e. a multiplication by $\sum \xi_{k}^{2}$. Hence both conditions of the theorem of Hamilton [3, Theorem 5.1] are satisfied, and the conclusion follows.

To prove the smoothness property of the flow (3.4) we follow closely the idea presented in [1]. Let $E \rightarrow M$ be a vector bundle with connection $D$ over a compact manifold $M$ and let $F^{D}$ be its curvature. The flow of connections defined by the equation $\frac{\partial}{\partial t} D=-\tilde{\delta}^{D} F^{D}$ implies the following heat equation for the curvature:

$$
\frac{\partial}{\partial t} F^{D}=-\Delta^{D} F^{D}
$$

where $\Delta^{D}=d^{D} \tilde{\delta}^{D}+\tilde{\delta}^{D} d^{D}$. By the Weitzenböck formula,

$$
\Delta^{D} F^{D}=\Delta F^{D}+K_{1}\left(F^{D}\right)+K_{2}\left(F^{D}\right)
$$

where $\Delta=-D_{i} D_{i}$ is the rough Laplacian, $K_{1}$ is linear in $F^{D}$ and involves the curvature of the base space, and $K_{2}$ quadratic in the curvature $F^{D}$. Let $g_{E}$ be a family of metrics on $E$ and assume that

$$
\left|\frac{\partial}{\partial t} g_{E}\right| \leq C
$$

THEOREM 4.2. There exist constants $C(q)$ and $\delta(q)$ depending on the dimension of $E$ only such that the equation (4.14) has a solution for any initial condition $F^{D}(0)$ with $\left|F^{D}(0)\right| \leq 1$ on the interval $[0, \delta(q)]$, and

$$
\sum_{k=0}^{\infty} \frac{t^{k}}{((k+1) !)^{2}}\left|D^{k} F^{D}\right|^{2} \leq C(q)
$$

The proof will be based on estimates given in the next few lemmas. By $C$ we denote any constant which depends only on the dimension of the manifold $M$ and the variance of the tensor $T$.

Lemma 4.3. For any tensor $T$

$$
\Delta|T|^{2}=2\langle\Delta T, T\rangle-2|\nabla T|^{2} .
$$

Lemma 4.4. Let $T$ be any tensor and $\Delta$ the rough Laplacian. Then

$$
\Delta\left(D^{k} T\right)-D^{k}(\Delta T)=\sum_{i=0}^{k} c_{i}\left(D^{i} F^{D}\right)\left(D^{k-i} T\right)
$$

where $c_{i} \leq\left(\begin{array}{c}k+2 \\ i+2\end{array}\right)$ for $0 \leq i \leq k$. 
Proof. We use the induction and the fact that

$$
\Delta\left(D^{k} T\right)=D\left(\Delta\left(D^{k-1} T\right)\right)-2 F^{D} \cdot D^{k} T-D F^{D} \cdot D^{k-1} T .
$$

Lemma 4.5. Assume that $\frac{\partial}{\partial t} D=-\tilde{\delta}^{D} F^{D}$. Then

$$
\frac{\partial}{\partial t}\left(D^{k} T\right)-D^{k}\left(\frac{\partial}{\partial t} T\right)=\sum_{i=1}^{k}\left(\begin{array}{l}
k \\
i
\end{array}\right)\left(D^{i-1}\left(\frac{\partial}{\partial t} D\right)\right)\left(D^{k-i} T\right)
$$

and hence

$$
\left|\frac{\partial}{\partial t}\left(D^{k} T\right)-D^{k}\left(\frac{\partial}{\partial t} T\right)\right| \leq C \sum_{i=1}^{k}\left(\begin{array}{c}
k \\
i
\end{array}\right)\left|D^{i} F^{D} \| D^{k-i} T\right|
$$

Combining lemmas 4.4 and 4.5 we obtain

LEMMA 4.6.

$$
\left|\left(\frac{\partial}{\partial t}+\Delta\right) D^{k} F^{D}\right| \leq C\left(\sum_{i=0}^{k}\left|D^{i} F^{D}\right|+\sum_{i=0}^{k}\left(\begin{array}{c}
k+2 \\
i+2
\end{array}\right)\left|D^{i} F^{D}\right|\left|D^{k-i} F^{D}\right|\right)
$$

For $m \geq 1$ we define

$$
\begin{gathered}
a_{k}=\frac{t^{k / 2}}{(k+1) !}\left|D^{k} F^{D}\right|, \quad \varphi=\sum_{k=0}^{m} a_{k}^{2} \quad \text { and } \\
b_{k}=\frac{t^{(k-1) / 2}}{k !}\left|D^{k} F^{D}\right|, \quad \psi=\sum_{k=1}^{m} b_{k}^{2} .
\end{gathered}
$$

LEMMA 4.7. For $m \geq 1$ there is the estimate

(4.26) $\sum_{k=0}^{m} \sum_{i=0}^{k} \frac{t^{k}}{((k+1) !)^{2}}\left(\begin{array}{c}k+2 \\ i+2\end{array}\right)\left|D^{i} F^{D}\right|\left|D^{k} F^{D}\right|\left|D^{k-i} F^{D}\right| \leq 2\left(t \varphi^{1 / 2} \psi+\varphi^{3 / 2}\right)$.

Assume that $t \leq 1$. Then

$$
\sum_{k=0}^{m} \frac{t^{k}}{((k+1) !)^{2}} \sum_{i=0}^{k}\left|D^{i} F^{D}\right|\left|D^{k} F^{D}\right| \leq 6 \varphi .
$$

Proof of theorem 4.2. Combining lemmas 4.3 and 4.6 with the uniform bound on the change of $g_{E}$ we obtain

$$
\begin{aligned}
& \frac{\partial}{\partial t}\left|D^{k} F^{D}\right|^{2} \leq C\left|D^{k} F^{D}\right|^{2}+C \sum_{i=0}^{k}\left|D^{i} F^{D}\right|\left|D^{k} F^{D}\right| \\
& +C \sum_{i=0}^{k}\left(\begin{array}{c}
k+2 \\
i+2
\end{array}\right)\left|D^{i} F^{D}\right|\left|D^{k} F^{D}\right|\left|D^{k-i} F^{D}\right|-\Delta\left|D^{k} F^{D}\right|^{2}-2\left|D^{k+1} F^{D}\right|^{2} .
\end{aligned}
$$


We then estimate $\frac{t^{k}}{((k+1) !)^{2}}\left|D^{k} F^{D}\right|^{2}$ using (4.28), add up the inequalities thus obtained (from $k=0$ to $m$ ) and combine with lemma 4.7 , obtaining the inequality

$$
\frac{\partial}{\partial t} \varphi \leq-\Delta \varphi-\left(1-C t \varphi^{1 / 2}\right) \psi+C \varphi^{3 / 2}+C \varphi
$$

The maximum principle applied to (4.29) together with the fact that (by the assumption) $|\varphi(0)|=\left|F^{D}(0)\right|^{2} \leq 1$, implies

$$
\varphi(t) \leq \frac{1}{\left(2 e^{-C t / 2}-1\right)^{2}}
$$

for all $t \in[0, T], T=\frac{1}{2 C} \leq 1$, and the theorem follows.

\section{REFERENCES}

[1] S. BANDO, Real analyticity of solutions of Hamilton's equation, Math. Z., 195 (1987), pp. 93-97.

[2] J. Bemelmans, M. Min-Oo, And E. A. RuH, Smoothing Riemannian metrics, Math. Z., 188 (1984), pp. 69-74.

[3] R. S. HAmilton, Three-manifolds with positive Ricci curvature, J. Differential Geom., 17 (1982), pp. 255-306.

[4] R. S. Hamilton, Four-manifolds with positive curvature opeartor, J. Differential Geom., 24 (1986), pp. 153-179.

[5] R. S. HAmilton, The Ricci flow on surfaces, Contemporary Mathematics, 71 (1988), pp. 237262.

[6] J. J. HEBDA, An example relevant to curvature pinching theorems for Riemannian foliations, Proc. of the AMS, 114:1 (1992), pp. 195-199.

[7] G. HuISKen, Ricci deformation of the metric on a Riemannian manifold, J. Differential Geom., 21 (1985), pp. 47-62.

[8] C. MARgerin, Pointwise pinched manifolds are space forms, in Proc. Summer Inst., Arcata 1984, Proc. Symp. Pure Math. 44, 1986, pp. 307-328.

[9] M. Min-Oo ANd E. A. Ruh, Curvature deformations, in Proc. Taniguchi Symp., Katata 1985, Lecture Notes in Math. 1201, Springer, Berlin, 1986, pp. 180-190.

[10] M. MiN-Oo, E. A. RUH, AND P. TONDEUR, Vanishing theorems for the basic cohomology of Riemannian foliations, J. Reine Angew. Math., 415 (1991), pp. 167-174.

[11] P. Molino, Riemannian Foliations, Birkhäuser, Boston, 1988.

[12] S. NishikaWA, Deformation of Riemannian metrics and manifolds with bounded curvature ratios, in Proc. Summer Inst., Arcata 1984, Proc. Symp. Pure Math. 44, 1986, pp. 343352.

[13] P. Tondeur, Foliations on Riemannian Manifolds, Springer, Berlin, 1988. 